\title{
Experimental Electric Power System for Small Satellites with Independent Supply Channels
}

\author{
${ }^{1)}$ Ales Vobornik, ${ }^{2)}$ Ivo Vertat, ${ }^{3)}$ Richard Linhart \\ Faculty of Electrical Engineering \\ University of West Bohemia \\ Pilsen, Czech Republic \\ 1)vobornik@ket.zcu.cz, ${ }^{2)}$ ivertat@kae.zcu.cz, ${ }^{3)}$ rlinhart@kae.zcu.cz
}

\begin{abstract}
Small satellites such as CubeSats are not adequately protected in the cosmic environment from ionizing radiation due to the low weight limit, which does not allow the use of heavy shielding materials with the high atomic number. Therefore, the power supply system of such small satellites must be protected from radiation induced failures in the satellite electronic subsystems. The power supply system must also provide a reliable power supply in the event of a fault in its own components. This requires more independent power supply channels for satellite subsystems and reliable protection circuits. In this paper, we propose an electric power system with three independent channels and necessary protection circuits for the PilsenCube II satellite. Two supply channels use Li-Ion accumulators and unregulated bus. The third supply channel uses supercapacitors and regulated bus. The three independent supply channels are complemented by the overcurrent protection circuits in each subsystem board that allow controlled post-fault regeneration.
\end{abstract}

Keywords - satellite electric power system; overcurrent protection circuit; latch up effect protection; single event protection; picosatellite; nanosatellite

\section{INTRODUCTION}

The traditional solutions of electric power systems (EPS) for small satellites differ in principle of the electric energy distribution from solar cells into energy storage devices and also in principle of its distribution to powered subsystems. There are many different solutions exist that are described in [1]. However, only a few basic topologies are commonly used in small satellites.

In the simplest form, the solar cell subsystem can be connected to an energy storage device and powered subsystems by the direct energy transfer method (DET) or the maximum power point tracking method (MPPT). Direct energy transfer connects solar cells directly to the energy storage device (e.g. accumulator) without the use of voltage converters. This method achieves low complexity and high reliability but reduces energy efficiency due to the load of solar cells at the non-optimal point of their current-voltage characteristics [2]. The maximum power point tracking method links solar cells with energy storage using controlled voltage converters. This method achieves higher energy efficiency due to the load of solar cells at optimum point of their current-voltage characteristics but reduces reliability, increases system weight and complexity of the system [3].

The subsequent distribution of electrical energy from solar cells and from energy storages to powered subsystems can be realized via a voltage-regulated supply bus [4] or an unregulated bus [2]. The voltage regulated distribution requires a centralized voltage converter (or more converters for more distributed voltage levels) instead of dedicated converters in individual satellite subsystems, which also reduces system weight and complexity. However, the centralized voltage converter also reduces reliability (its failure affects multiple powered subsystems) and efficiency (the centralized converter must be designed for peak power consumption, but will work at a lower load for most of the time). Unregulated power distribution directly from solar cells and energy storage devices requires in most subsystems their own voltage converters, which increases the complexity and weight of system. On the other hand, this solution increases reliability (the converter failure affects only one powered subsystem) and increases energy efficiency (dedicated converters can be properly dimensioned according to the requirements of the individual subsystems). Another advantage is that many subsystems do not require converters due to their ability to fully operate over a wide range of unregulated voltage level.

Our proposed EPS system is a hybrid of the above methods. Our goals is to provide a simple and reliable supply of satellite subsystems as well as to safely test the new supercapacitor technology in the satellite power system. The rest of the paper is organized as follows. Section II describes the topology of proposed electric power system, including solar panels and used energy storage devices. Section III presents the hardware solution for three independent supply channels that provide electricity to the satellite subsystems. In section IV, there are described the protection circuits located on individual boards of the satellite subsystems.

\section{BASIC TOPOLOGY OF PROPOSED EPS}

The electric power system is designed as redundant with three independent supply channels. Each supply channel has its own solar panels, energy storage and a bus for the distribution of energy to the satellite subsystems. All supply channels are partially 
protected against overload. The merging of supply channels and other protecting circuits are located on each powered boards.

Two independent supply channels are designed to be reliable with low complexity. These supply channels are constructed from components tested by Cobalt-60 ionizing radiation source up to $30 \mathrm{krad}$ of the total ionizing dose. Direct energy transfer to LiIon accumulators is used in these two channels and power is distributed to satellite subsystems via two unregulated power buses. Rated voltage of used accumulators and used solar cells ensure that solar cells work close to the point of maximum power, which reduce the efficiency loss of DET method. The long-term reliability of the power supply of the satellite is the main purpose of these two supply channels.

The third supply channel is experimental and includes a MPPT converter between solar cells and supercapacitors and a digitally controlled converter between supercapacitors and powered subsystems. Not all of the components in this channel are tested by a source of ionizing radiation. The main purpose is to experimentally test the possibilities of extending accumulator lifetime by controlled release of energy stored in the supercapacitors for powering the satellite when the accumulators are in unsuitable conditions (low temperature, deep discharge). The contribution from the third channel to the total power of the satellite is continuously regulated by adjusting the output voltage of this supply channel. All three channels are merged and protected against overcurrent by LTC4411 on each individual subsystem board. The merging and protection circuits are also constructed from the radiation tested components as the first two supply channels.

Three independent supply channels are powered by six solar panels [5] located on all the walls of the cube-shaped satellite body. All solar panels have a parallelized and redundant topology, using up to 24 triple junction $\mathrm{GaInP}_{2} / \mathrm{GaAs} / \mathrm{Ge}$ small solar cells per solar panel. These small solar cells are manufactured by Spectrolab Company as a TASC product (triangular advanced solar cell). One solar panel is made up of up to four independent basic blocks of solar cells that are merged with the LTC4411 or MBR120VLSF devices. This structure of solar panels is highly tolerant to failure of individual solar cells, which potentially affects only a small part of the solar panel area. A large number of small solar cells on one solar panel also allows for any serial/parallel combination of cells with output voltage adapted to the needs of energy storage device or integrated step up/down converters. As shown in fig. 1 and fig. 2, we use a serial connection of two solar cells into the basic unit. Several basic units can be connected in parallel to increase the output current (used in supply channel 1 and 2) or in series to increase the output voltage (used in supply channel 3).

Always two solar panels on the opposite sides of the satellite supply energy to one of three independent channels, each equipped with its own energy storage. Two of the three supply channels use the Li-Ion accumulators Panasonic NCR18650B-U (one per supply channel) in a cylindrical package 18650 and a nominal capacity of $3400 \mathrm{mAh}$. The third supply channel utilizes the serial connection of two Maxwell BCAP0350 supercapacitors with a nominal capacity of $350 \mathrm{~F}$ and a nominal voltage of $2.7 \mathrm{~V}$. Two used Li-Ion accumulators and two supercapacitors allow to store a total energy of approximately $25 \mathrm{Wh}$. Such amount of storable energy is capable of delivering high demands on the power of the satellite subsystems even when flying in the shadow of the Earth.

The complete wiring diagram of the proposed power supply is too complicated for the presentation and detailed description to a limited extent of this article. However, the most important parts, including the three main supply channels and many auxiliary and protective circuits, will be described in the following sections.

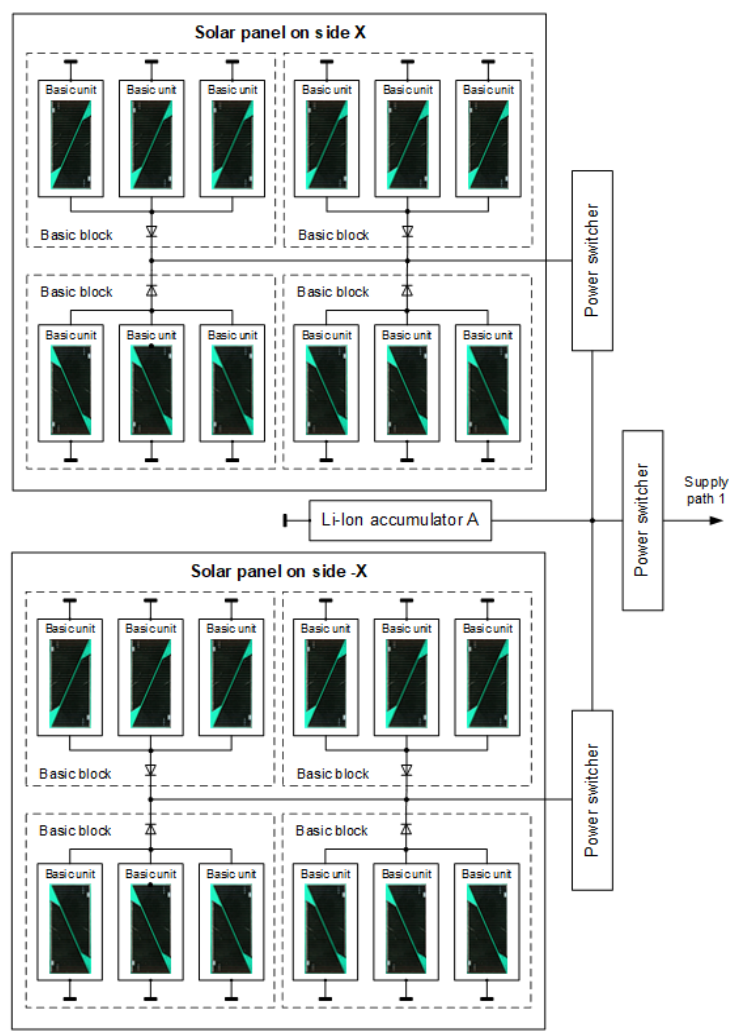

Figure 1. Simplified block diagram of a conventional power supply channel 1 with a parallel and redundant structure of solar panels optimized for direct energy transfer to Li-Ion acumulators.

\section{PROPOSED SUPPLY CHANNELS}

There are two conventional and one experimental supply channels in the PilsenCube II satellite. Two conventional channels use direct energy transfer, LiIon accumulators and a voltage unregulated supply buses (channel 1 and 2). The experimental supply channel uses an input MPPT converter, two supercapacitors connected in series and an output converter with digitally controlled output voltage (channel 3). 


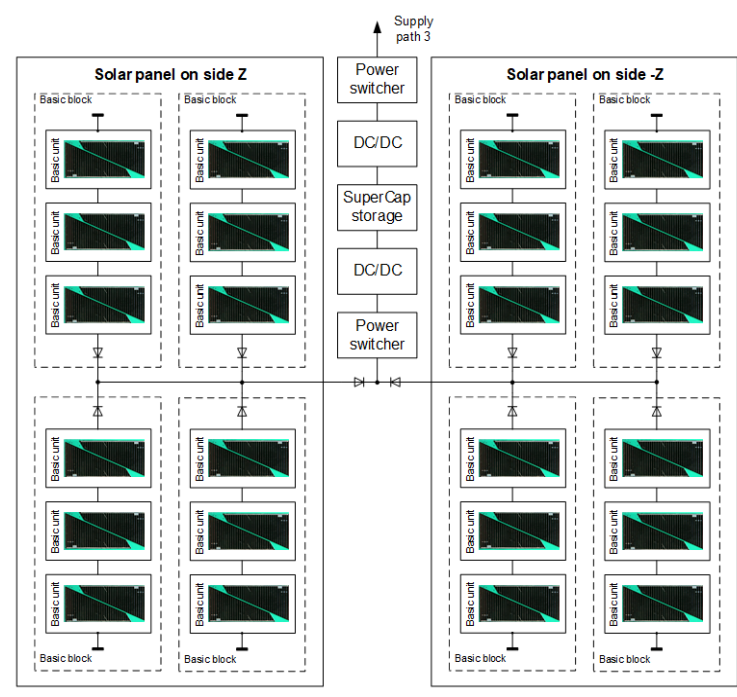

Figure 2. Simplified block diagram of experimental supply channel 3 with parallel and redundant solar panel structure optimized for maximum power point tracking converter.

Each supply channel is equipped with a power switch, overvoltage and undervoltage protection and overcurrent resettable fuse to protect energy storages from overloading. The three supply channels are distributed separately to satellite subsystems in which the channels are merged through LTC4411 circuits. All subsystems have implemented an overcurrent protection of the merged power supply and an automatic undervoltage control is performed in most minor subsystems, which disconnects them in case of low voltage from the supply channels.

The proposed EPS is equipped with the STM32F100 processor for controlling the experimental supply channel 3 and for on-board diagnostics of the entire power supply system. Supply channels 1 and 2 are completely independent of the correct functionality of the EPS processor and the supply channel 3 can also operate without active processor control where the converter output voltage remains set according to the last configuration of the digital potentiometer.

\section{A. Conventional Channels with Li-Ion Accumulators}

The simplified block diagram of the conventional supply channel 1 is shown in fig. 1. This channel utilizes two solar panels on opposite sides $\mathrm{X},-\mathrm{X}$ and one of two Li-Ion accumulators. The supply channel 2 is identical to channel 1 , only uses $\mathrm{Y},-\mathrm{Y}$ solar panels instead of $\mathrm{X}, \mathrm{X}$ and uses a second accumulator. These two conventional supply channels are complemented by several support circuits that are needed to better diagnose the EPS system, protect it against failure and overloading, and also meet the CubeSat design specifications [6] for their electrical systems. In particular, it is necessary to ensure complete deactivation of the EPS system during the integration of the satellite into the orbital deployer until the satellite is released into the orbit. One of the additional circuits in supply channel 1 and 2 is the power switch and the current sensor at the channel input, shown in fig. 3 .

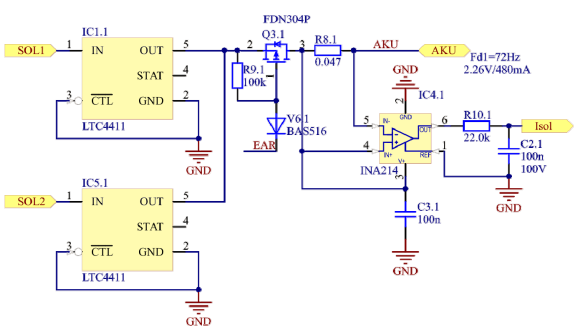

Figure 3. Two power switches and current sensing operational amplifier at the input of conventional supply channel 1 (or 2).

The two LTC4411 power switches are used to safely merge two solar panels $\mathrm{X}$ and $-\mathrm{X}$ in the supply channel 1 (or $\mathrm{Y}$ and $-\mathrm{Y}$ in case of the supply channel 2 ). The transistor FDN304P is used to disconnect the merged solar panels from the energy storage and from subsystems during the satellite integration into rocket as required by the CubeSat design specification [6]. The INA214 operational amplifier is used to measure the current of solar panels, useful for the onboard diagnostics of EPS system.

Another additional circuit consists of power switches and a current sensing amplifier at the output of a conventional supply channel 1 (or channel 2), shown in fig. 4. The two STMPS2171 switches are used to disconnect the energy storage and solar panels from the powered subsystems as required in [6]. The second function is to protect the energy storages from overloading due to short-circuit or abnormal power consumption in powered subsystems. In normal mode, only one of the two STMPS2171 switches is active and provides a supply of a maximum of $1 \mathrm{~A}$ to the powered subsystems via supply channel. If necessary, the EPS system processor can enable power up to $2 \mathrm{~A}$ by activating the second STMPS2171 switch. The output current of supply channel is measured by the INA214 operational amplifier for better onboard diagnostics.

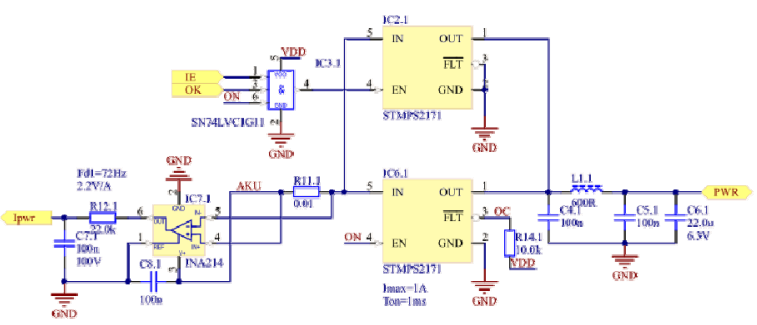

Figure 4. Two power switches and current sensing operational amplifier at the output of conventional supply channel 1 (or 2).

Other important supporting circuits are undervoltage and overvoltage protection of accumulators in conventional supply channels that prevent deep discharging or overcharging of Li-Ion accumulators. These protections are realized by two comparators OPA2330 shown in fig. 5 and fig. 6. The undervoltage limit for the load disconnection is set to $3.27 \mathrm{~V}$ with small hysteresis (reataching of the load from $3.33 \mathrm{~V}$ ). The overvoltage condition may occur rarely due to the characteristics of solar cell voltage, its temperature dependence and the voltage drops on merging diodes and switches. Therefore, the overvoltage protection is realized only by reporting to 
the main control processor of the satellite and activating the additional power consumption, which leads to a voltage drop in conventional supply channels.

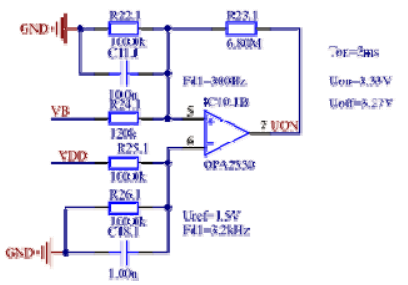

Figure 5. The operational amplifier as comparator with hysteresis for controling of undervoltage condition of Li-Ion accumulators.

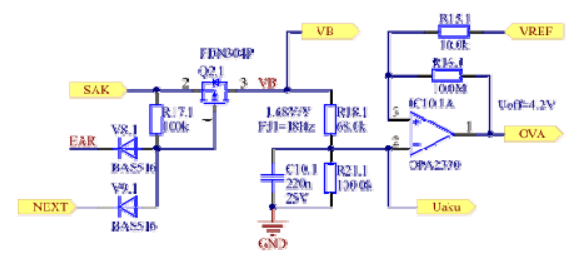

Figure 6. The operational amplifier as comparator for controling of overvoltage condition of Li-Ion accumulators.

\section{B. Experimental Channel with Supercapacitors}

The simplified block diagram of experimental supply channel 3 is shown in fig. 2. This channel utilizes two solar panels on opposite sides $Z,-Z$ and two supercapacitors in series as an energy storage. This experimental supply channel uses an input DC/DC converter controlled in MPPT mode and the output converter controlled by the EPS processor for supplying more or less power as a support for two conventional supply channels. Supply channel 3 is also complemented by several support circuits that are needed to better diagnose the EPS system and to completely deactivate the EPS system during the integration of the satellite into the orbital deployer.

Fig. 7 shows a solar panel $(Z,-Z)$ merging circuit made of the discrete diode MBR120VLSF, followed by power switch from the FDN304P transistor and a current sensing operational amplifier INA214.

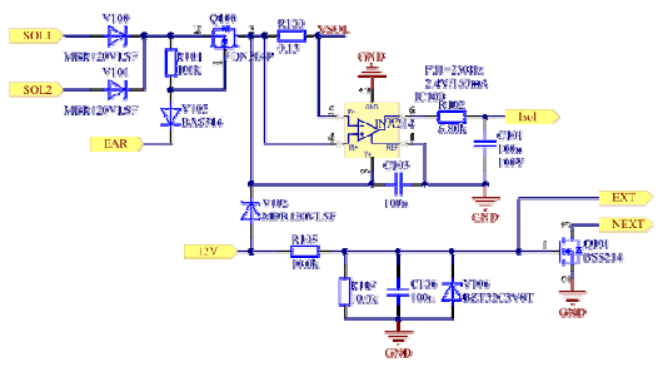

Figure 7. Merging diodes, the power switch and the current sensing operational amplifier at the input of supply channel 3.

The integrated charging circuit LT1510 is used as an input DC/DC converter that supplies energy into two series-connected supercapacitors. The programming input of the LT1510 serves to control the charging current of supercapacitors, thus enabling the implementation of the MPPT principle. The programming input is controlled by the EPS processor using the digital potentiometer AD5161.

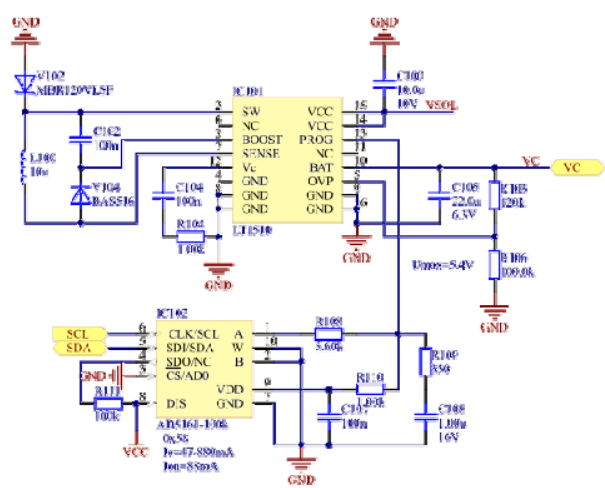

Figure 8. The input DC/DC converter in channel 3, working in MPPT mode and controled by the EPS processor via digital potentiometer.

Power supply of the satellite subsystems from two supercapacitors is realized via the output DC/DC converter TPS63020 (fig. 9). The start of this converter is delayed until the supercapacitors are charged atleast to $1.85 \mathrm{~V}$. This sequenced start is performed by the MAX6895, shown in fig. 10. The output voltage of the second converter (output voltage of channel 3) is controlled by the EPS processor via the digital potentiometer AD5161 in the range of $3.4 \mathrm{~V}$ to $4.9 \mathrm{~V}$. With this output voltage regulation, the supply channel 3 can také more or less power supplying to the satellite subsystem through the merged channels 1, 2 and 3, depending on the actual charge of the accumulators and supercapacitors.

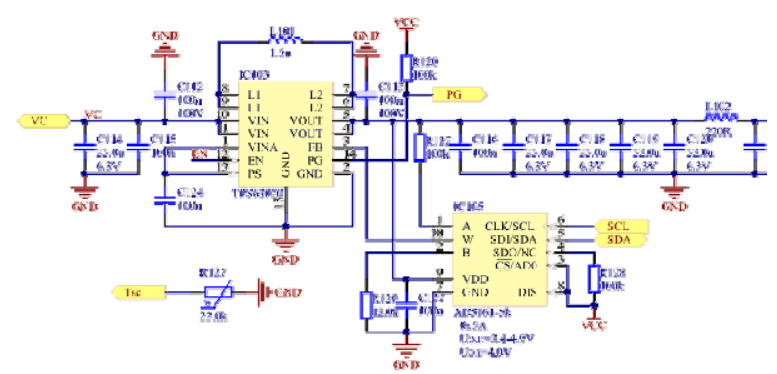

Figure 9. The output DC/DC converter in channel 3, controled by the EPS processor via digital potentiometer.

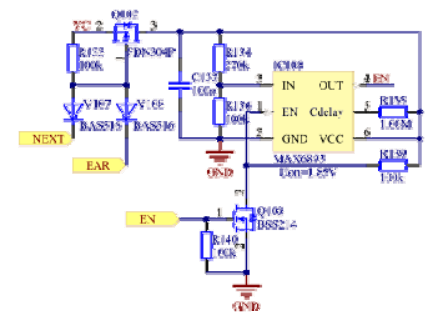

Figure 10. Circuit MAX6895 for the sequenced start of two

DC/DC converters in the experimental supply channel 3.

The output of supply channel 3 is protected against overcurrent above 1 A by the STMPS2171 switch (fig. 11), which must by deactivated when integrating the satellite into the deployer and also deactivated if the output voltage of the TPS63020 is less than $3.34 \mathrm{~V}$ due to any failure of the supply channel 3. 


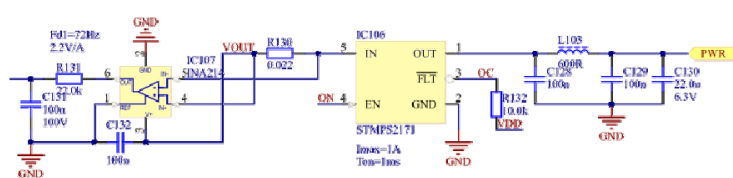

Figure 11. The power switch at the output of supply channel 3 with the current sensing operational amplifier INA214.

\section{Processor Part of Supply Channels}

The processor part of the EPS system with the STM32F100 (fig. 12) has only supporting tasks and the EPS system is capable of functionality even when the processor fails. The main purpose of the EPS processor is to collect voltage and current measurements from all three supply channels and report the status of stored energy in accumulators and supercapacitors to the main satellite processor for better planning of energy-intensive experiments. Other tasks of the processor are to control the digital potentiometers for implementing the MPPT mode of the input converter and balancing the power supply from the regulated channel 3 and the unregulated channels 1 and 2. In case of the EPS processor failure, the both digital potentiometers remain in the last configuration and the channel 3 is still capable of delivering the power, but not in the optimal mode.

The processor is only required during the initial phase of the satellite operation for self-initialization of antenna deployment or, in the event of its failure, for initializing the antenna deployment by a command from the ground station. Therefore, the EPS processor was also tested on a source of ionizing radiation as all the components used in supply channel 1 and 2 .

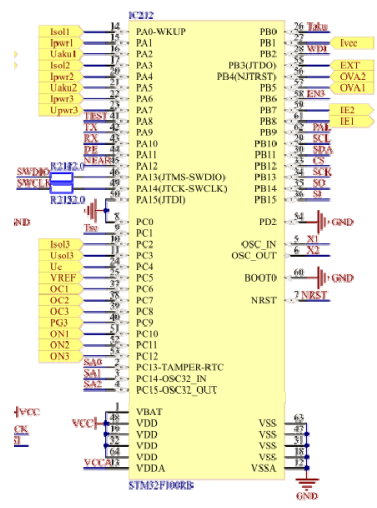

Figure 12. Processor of the EPS for the system onboard diagnostics via analog inputs and for an active control of the supply channel 3 .

\section{PROTECTION CIRCUITS ON SUPPLIED BOARDS}

The previously described switches STMPS2161 and STMPS2171 used in individual supply channels are designed to prevent total EPS overload in the event of unintentional parallel operation of high power demanding subsystems. However, it does not solve the protection of the powered subsystems by quickly interrupting their overcurrent and preventing the continuous depletion of the source by faulty subsystems. Therefore, the concept of the proposed EPS system includes several other protection circuits, which are obligatory placed on the individual boards of subsystems. These circuits control the correct supply of subsystems, prevent high current peaks in the supply channels and enable selective deactivation of subsystems according to the actual voltage in case of unintentional power shortages in supply channels.

One of the protection circuits in the powered subsystems is shown in fig. 13. This circuit with three LTC4411 devices merges the three independent supply channels.

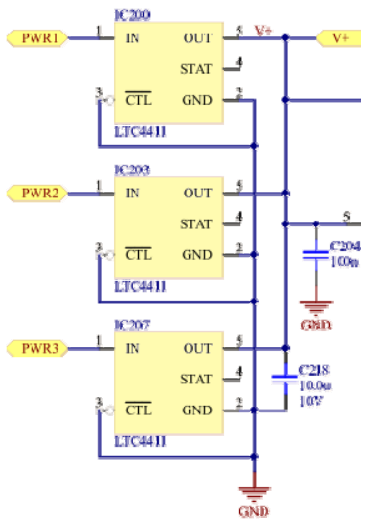

Figure 13. Circuit for the current protection and merging of supply channels placed in all powered boards.

The voltage of the merged channels is monitored by the operational amplifier OPA2330, which is used as a comparator with hysteresis (fig. 14). The purpose of this circuit is to generate a signal to disconnect the subsystem if the input voltage is lower than preset value. Important subsystems (radio, main onboard computer) must be disconnected from the power supply at a voltage of less than $3.3 \mathrm{~V}$ on the merged supply channels output, less important subsystems must be disconnected already when the voltage falls below $3.8 \mathrm{~V}$. This ensures that only the most important systems remain in operation and less important systems are automatically shut down in the event of power shortages.

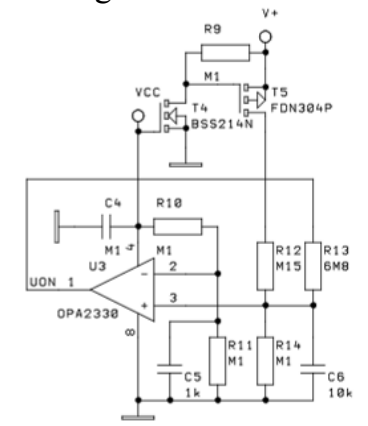

Figure 14. Circuit for the selective undervoltage deactivation of less important subsystems in the case of power shortages.

This undervoltage control circuit is powered from a small linear low-drop voltage regulator (fig. 15) with a delayed start in the range of $2 \mathrm{~ms}$ to $8 \mathrm{~ms}$ after the supply is applied to the input. This adjustable delay determines the subsystem's awakenings, so the subsystems do not start working at the same time when the power supply is restored. This limits unwanted current peaks in supply channels and potential risks of system instability. 


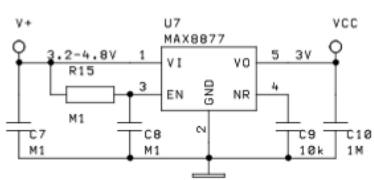

Figure 15. Low-drop voltage regulator for a delayed supply of the protection circuits and delayed sequenced starts of all subsystems.

The most important parts of the protection circuits located on the powered boards are current sensors with power switches (fig. 16), current limiters (fig. 17) and the watchdog timers (fig. 18). Current measurement with the LT6105 amplifier allows to set several current limits by changing the sensitivity of current measurement. In the OPA2330, the measured current is compared to the threshold and in the case of overcurrent detection, the supply of subsystem is interrupted by the STMPS2151 switch.

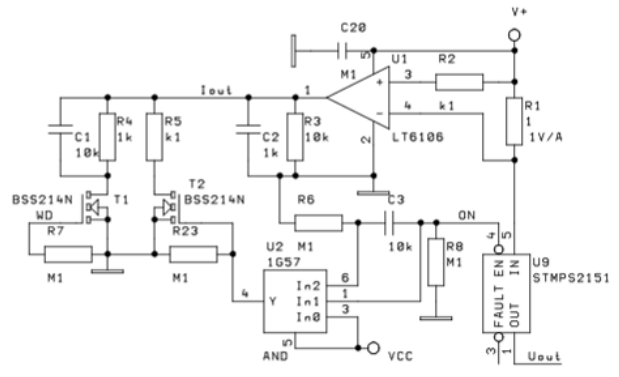

Figure 16. The current sensing operational amplifier with the power switch at the input of powered subsystems.

The switching of the transistor T2 in fig. 16 allows a short peak current draw without activation of the overcurrent protection after startup of the supply for the charging the blocking capacitors in the subsystems. The switching of the transistor T1 allows the allocation of a full current limit after the watchdog timer is periodically serviced by the subsystem processor. Without switching on the T1 and T2 transistors, the subsystems only get the low current limits necessary to start the subsystem processor.

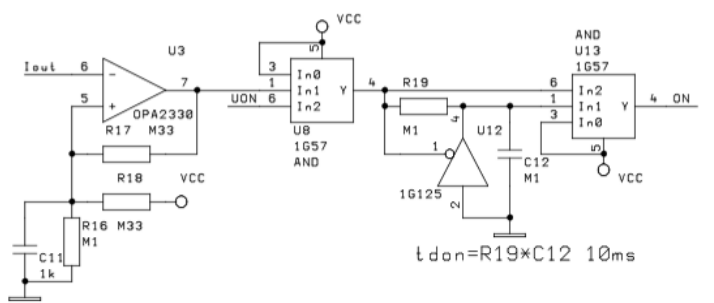

Figure 17. The operational amplifier for comparing the measured input current with the treshold for an overcurrent indication.

If the subsystem processor fails, the watchdog circuit (fig. 18) timeout is exceeded. This causes the current limit to be reduced and the STMPS2151 temporarily interrupted the supply by overcurrent detection. The switch is reactivated with a fixed time delay after overcurrent detection and the subsystem has only a low current limit to run the processor. If the watchdog timer starts to be periodically serviced by the processor, the subsystem receives a full current limit and the subsystem processor receives full access to the main data bus via the LTC1400.

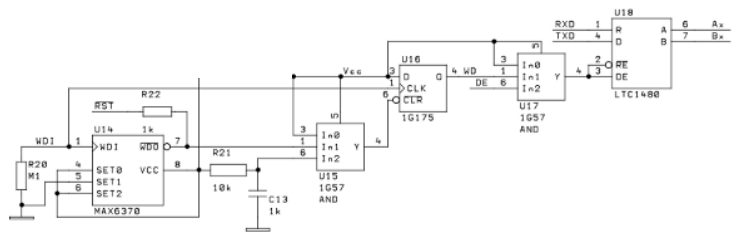

Figure 18. The watchdog timer for an active control of subsystem current limit and the subsystem processor access to the data bus.

\section{MECHANICAL SOLUTIONS OF EPS}

The mechanical design of the PilsenCube II satellite without solar panels and without a few other subsystems is shown in fig. 19. This model shows the main boards of the satellite system (electric power supply system, measuring system, onboard data handling and main radio communication system, secondary radio communication system, pixel based particle detector system) and a block of energy storages.

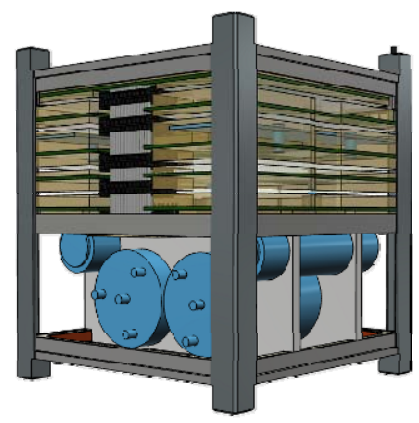

Figure 19. Two supercapacitors BCAP0350 and two Li-Ion accumulators NCR18650B-U (blue colors) in PilsenCube II.

Energy storages (two accumulators and two supercapacitors) are located at the bottom part of the satellite and there are mechanically held in PEEK material plates. PEEK material is a type of plastic suitable for space applications with good mechanical properties. PEEK plates also heat-insulate the accumulators, which is good for eliminating their low temperature during satellite flight in the eclipse. The rest of the free space around the energy storage block is used for other satellite systems like onboard camera, optical sensors, PIN diode detectors and service module (not shown in fig. 19). The EPS board is approximately in the middle of the satellite.

\section{CONLUSIONS}

The failures of satellite systems caused by cosmic radiation must be taken into account in the design of small satellites due to the limited possibilities of shielding this ionizing radiation. In this paper, we proposed a hardware solution for a simple electric power supply system for small satellites such as CubeSat. The proposed EPS system has three independent supply channels with its own energy storage and its own solar panels. Two supply channels are constructed only from components tested up to $30 \mathrm{krad}$ of total ionizing dose from Cobalt- 60 radiation source. This approximately corresponds to a dose of typical CubeSat missions (shielded with $1 \mathrm{~mm}$ thick aluminum plates) after two or three years on polar 
LEO orbits. The third experimental channel also uses untested components. The main purpose of the third supply channel is to test the capability of using supercapacitors in a small satellite electric power system, as conventional Li-Ion accumulators are not suitable for a long-term space mission. Our EPS system solution utilizes a number of protection circuits in supply channels and also mandatory in the powered subsystems, because most components in the subsystems are not tested for the effects of ionizing radiation. The radiation tests of modern electronics components are very time consuming and costly, and so many small satellite teams do not do them at all. However, thanks to the satellite system topology measures already made during the hardware design, several temporary or permanent failures in the satellite subsystems as well as in own components of the EPS system should not completely destroy the function of our satellite.

\section{ACKNOWLEDGMENT}

This project was funded by the Czech Science Foundation and registered as a project number
102/09/0455: Power efficient space probe for experimental research based on picosatellite.

\section{REFERENCES}

[1] G. Farahami, M. Taherbaneh, "Extracting best reliable scheme for Electrical Power Subszstem (EPS) of satellite" in Proceedings of 5th International conference on Recent Advances in Space Technologies - RAST2011, 2011, Pages 532-537, ISSN 0094-5765. doi: 10.1109/RAST.2011.5966894

[2] T. M. Lim, "A modular electrical power system for small spacecraft", Theses and disertations, University of Kentucky, 2016. doi: 10.13023/ETD.2016.331

[3] L. E. Jacobsen, "Power system on the NTNU test satellite", Technical report, Norwegian University of Science and Technology, 2011.

[4] R. P. Faria, C. P. Gouvea, J. C. V. de Castro, R. Rocha, "Design and implementation of a photovoltaic system for artificial satellites with regulated DC bus." In IEEE $26^{\text {th }}$ International Symposium on Industrial Electronics. 2017. Pages 676-681. doi: 10.1109/ISIE.2017.8001327

[5] I. Vertat, A. Vobornik, "Efficient and reliable solar panels for small Cubesat picosatellites," In International Journal of Photoenergy, 2014. doi: 10.1155/2014/537645

[6] S. Lee, R. Munakata, "CubeSat design specification, revision 12," Technical specifications. 2009. 